\title{
Application of IR spectroscopy to control oxidation inhibitor (ionol) concentration in mineral transformer oils
}

\author{
Marsel Sh. Garifullin ${ }^{1 *}$, Marina N. Lyutikova ${ }^{2}$, Adelya R. Kuchkarova ${ }^{3}$, Azat R. Bikzinurov ${ }^{1}$, and Yuliya N. Solobodina ${ }^{1}$ \\ ${ }^{1}$ Kazan State Power Engineering University, Department of Electric Power Systems and Networks, 420066 Kazan, Krasnoselskaya str., \\ 51, Russia \\ ${ }^{2}$ Yamalo-Nenetsky PMES - branch of Federal Grid Company of Unified Energy System, Chemical laboratory diagnostic service, 629800 \\ Noyabrsk, Enthusiasts str., 10, Russia \\ ${ }^{3}$ EC «Energoprogress», Department of electrical equipment, 420080 Kazan, Volgogradskaya, str., 34, Russia
}

\begin{abstract}
Mineral insulating oils used as dielectric and heat dissipating agent in high voltage oil-filled equipment are subject to oxidation. Oxidation results in the appearance of undesirable substances that reduce the electrical insulating properties of liquids. Therefore, to inhibit the oxidation process, an antioxidant additive is introduced into transformer oils. In the overwhelming majority, 2,6-di-tert-butyl-4methylphenol (ionol) is used as an inhibitor. Residual concentration of ionol is a regularly monitored parameter. In Russia, the main method for determining the concentration of ionol in oil is gas chromatography. However, fourier-transform infrared spectroscopy should be considered as an alternative method for monitoring the ionol content as it has a number of advantages over the gas chromatographic method. The paper compares the results of the determination of ionol in operational transformer oils using gas chromatography and the IR method. We justified that the method of IR spectroscopy has no limitations as applied to oils from power transformers equipped with adsorption purification systems.
\end{abstract}

\section{Introduction}

Power transformers are among the most important and expensive equipment types in any power system. Considering that the average age of power transformers has long exceeded the established deadlines [1, 2], ensuring the reliability of their operation is one of the most important operational tasks.

The possibility of further operation of transformer is determined by the state of its insulation. Exposure to high temperature, electric field and other operating factors inevitably leads to aging of the insulation system. With regard to power oil-filled transformers, which make up the bulk of the entire transformer fleet, degradation of insulating paper and mineral (petroleum) transformer oil is implied.

In this case, it is necessary to note two important circumstances. First, the paper insulation of the transformer, unlike oil, cannot be replaced without a complex overhaul. That is, the unsatisfactory condition of the paper insulation means the exhaustion of the operational life of the transformer.

Second, the aging products of mineral oil accelerate the destruction of paper. Based on these provisions, it becomes obvious that a decrease in the rate of degradation of transformer oil makes it possible to extend the life and reliability of the transformer as a whole.

The main reason for the aging of transformer oil during its operation is the oxidation process. To slow down this process, special additives known as oxidation inhibitors are added to the oils. In Russia, as in many other countries, the most common oxidation inhibitor is ionol (2,6-di-tert-butyl-4-methylphenol). Ionol has many other trade names such as Agidol-1, 2,6-di-tert-butyl-p-cresol (DBPC), butylated hydroxytoluene (BHT), etc. Fig. 1 shows the structural formula of the ionol molecule and its 3D model.<smiles>Cc1cc(C(C)(C)C)c(O)c(C(C)(C)C)c1</smiles>

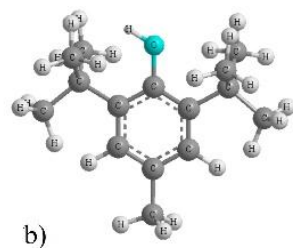

Figure 1. Structural formula (a) and 3D model (b) of the ionol molecule.

In the 80 s of the 20th century, Russian scientists Lipstein and Shakhnovich [3] found that to stabilize the oxidation processes the concentration of ionol should not decrease below $0.1 \%$ by weight. Russian regulatory documents set the concentration of ionol in transformer oils in the range of $0.1-0.40 \%$ [4]. In foreign standards, the minimum permissible ionol concentration is set at the level of $0.08 \%$ [5]. Thus, one of the operational tasks is the regular monitoring of the residual ionol content in insulating oils. Constant control makes it possible to promptly adjust ionol concentration to the required level.

* Corresponding author: g_marsels@mail.ru 
To solve the problem of control various chromatographic and spectral methods are used [6]. In Russian diagnostic laboratories, the most widespread method is gas-liquid chromatography (GLC) with a flame ionization detector (FID) [7]. The main disadvantages of all chromatographic methods are the long duration and high labor intensity of the analysis, difficulties with the supply of highly purified rectified ethanol with a volume fraction of alcohol of at least $96 \%$, which is used for ionol extraction.

An alternative method for determining the concentration of ionol is the IR spectrophotometric method. The analysis methodology is presented in documents [8, 9]. The use of modern IR spectrophotometers with Fourier transform makes it possible to significantly reduce the labor intensity, cost and duration of analysis in comparison with gas chromatography. In addition, the spectral method makes it possible to limit the use of chemical reagents. This is very important for the safety and health of personnel in chemical laboratories.

The only drawback of the IR method, which can hold back its widespread introduction into everyday practice, is the error in the analysis of highly oxidized oil samples. It is generally accepted that the results of the analysis give an overestimated value [10] when developing a calibration equation for one absorption band in the range of $3650 \mathrm{~cm}^{-1}$. The intensity of this absorption band, related to the stretching vibrations of the $-\mathrm{OH}$ group of the ionol molecule, is directly proportional to the total content of the additive in the oil.

The reason for the error in measurements by the IR method is that during the oxidation process in the oil, not only decomposition products of the ionol molecule are accumulated. It is possible that other compounds with the same absorption band in the range of $3650 \mathrm{~cm}^{-1}$ will be accumulated. The superposition of these absorption bands on the absorption band of ionol leads to distortion of the analysis results.

At the same time, it is necessary to pay attention to a certain regularity revealed during the analysis of a large number of oil samples from oil-filled electrical equipment of various types. The tendency of the accumulation of compounds in the oil which are decomposition products of the ionol additive, which affect the results of ionol measurements, is primarily characteristic of measuring current and voltage transformers [11]. For oils used in power transformers, such a process of formation of ionol oxidation products is rarely observed.

In this regard, it is interesting to compare the results of determining the concentration of ionol in oils from power transformers obtained by the traditional method of GLC and by the method of FTIR spectroscopy.

\section{Experimental part}

For the study, we used samples of transformer oils from 29 power transformers with a rated higher voltage from 110 to $500 \mathrm{kV}$.
The ionol concentration in the oil samples was determined by the GLC method according to [7], as well as by the IR spectra of the oils. Chromatographic studies were carried out in two independent certified chemical laboratories.

The Tensor-27 (Bruker) FTIR spectrometer was used to record the IR spectra of oils. The spectra were recorded in the range $4000-3000 \mathrm{~cm}^{-1}$ with a spectral resolution of $1 \mathrm{~cm}^{-1}$. The cuvette thickness was $2 \mathrm{~mm}$. The material of the cuvette windows was $\mathrm{CaF}_{2}$.

The certified reference materials (CRM 10203-2013) with ionol concentrations of $0.1 \%, 0.2 \%$ and $0.4 \%$ were used to calibrate the IR spectrometer.

\section{Results and discussion}

The spectrometer was calibrated based on the measured CRM spectra. Fig. 2 shows the IR spectra of CRM samples with different ionol concentrations. The analytical band is the absorption band with a peak at $3650 \mathrm{~cm}^{-1}$ [12].

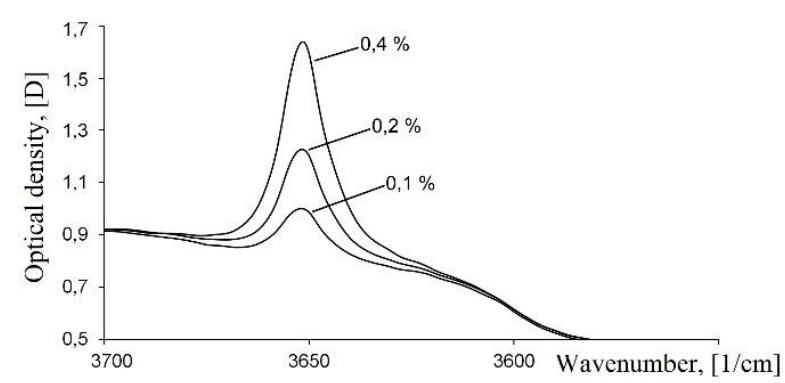

Figure 2. IR spectra of CRMs with different ionol content for cuvette thickness is $2 \mathrm{~mm}$.

The calibration curve of the ionol concentration in oil and the intensity of the absorption band is a linear regression shown in Fig. 3. The determination coefficient $R^{2}$ was 0.999 . The standard deviation was $0.002 \%$ of ionol. The result obtained allows us to assert that the using of a $2 \mathrm{~mm}$-thick cuvette ensures high measurement accuracy. At the same time, labor intensity and sample preparation time are reduced in comparison with the use of thinner cuvettes.

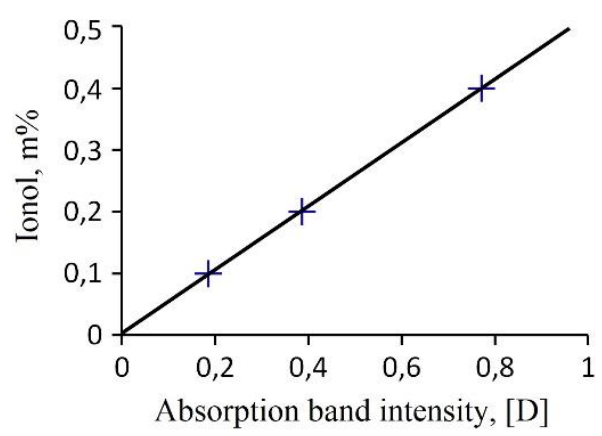

Figure 3. Calibration curve for determining the ionol concentration from the intensity of the absorption band near $3650 \mathrm{~cm}^{-1}$.

At the next step the spectra of all 29 oil samples were recorded. We determined the ionol concentrations in all oil samples from the intensities of the analytical 
absorption band in the region of $3650 \mathrm{~cm}^{-1}$ using the calibration equation. Table 1 shows the values of ionol concentration, obtained by the IR method, as well as the results of GLC measurements in two independent laboratories. For clarity, the same data are shown in Fig. 4 in the form of a bar graph: left columns - IR analysis; medium - Laboratory 1; right - Laboratory 2.

Analysis of the bar graph and tabular data shows that the results obtained by the IR analysis are consistent with those obtained by the gas chromatography. For most oil samples, the coefficient of variation of the results obtained by two independent laboratories and the IR method is less than $10 \%$. Thus, dispersion of data is insignificant.

The coefficient of variation $(\mathrm{CV})$ of more than $10 \%$ was obtained for four samples of oils (lines 6, 9, 11 and 23 in Table 1). Analysis of the data suggests that such a result was obtained in consequence of obviously erroneous measurements (faults) in Laboratory 1 (highlighted in grey in Table 1). In this regard, it is necessary to give explanations about the possible reasons for this kind of error.

When using GLC, erroneous measurements can occur if certain rules are not followed. In particular, in [13], the emergence of a significant methodological error in the case of excessive moisture of the extractant (ethyl alcohol) was demonstrated. Paper [13] also shows that the structural-group composition of the oil has a significant effect on the distribution coefficient of the inhibitor in the oil-ionol-alcohol system. It should be noted that this fact is not considered in the approved measurement procedures.

There is a problem, as in the case of IR spectroscopy, of overestimating the GLC results when ionol decomposition products appear in the oil, the concentration of which is calculated as a total [11].

Finally, there is the possibility of a blunder. To eliminate this kind of errors, measurements must be performed twice [7]. However, in practice, a single measurement is often used, which reduces the time and cost of analysis.

Thus, it can be stated that for the GLC method there is a problem of reproducibility of the results of ionol concentration measurement in insulating oils. Unlike gas chromatography, FTIR spectroscopy results practically do not depend on the qualifications of personnel and other subjective factors. Based on the analysis of the tabular data, we can conclude that the results obtained in
Laboratory 2 are more reliable. Therefore, we will further compare the results obtained by the IR spectroscopy method with the data of this laboratory.

Table 1. Results of ionol concentration measurement in transformer oils by IR spectra and gas-liquid chromatography.

\begin{tabular}{|c|c|c|c|c|}
\hline \multirow{2}{*}{ No. } & \multirow{2}{*}{$\begin{array}{c}\begin{array}{c}\text { Ionol } \\
\text { forecast }\end{array} \\
\text { IR }\end{array}$} & \multicolumn{2}{|c|}{ GLC } & \multirow{2}{*}{$\mathrm{CV}$} \\
\hline & & Lab.1 & Lab. 2 & \\
\hline 1 & 0,2343 & 0,2150 & 0,2363 & 4,2 \\
\hline 2 & 0,2651 & 0,2300 & 0,2658 & 6,6 \\
\hline 3 & 0,2362 & 0,2250 & 0,2343 & 2,1 \\
\hline 4 & 0,2534 & 0,2270 & 0,2551 & 5,2 \\
\hline 5 & 0,2545 & 0,2200 & 0,2785 & 9,6 \\
\hline 6 & 0,2731 & 0,2050 & 0,2609 & 12,0 \\
\hline 7 & 0,2617 & 0,2770 & 0,2595 & 2,9 \\
\hline 8 & 0,2532 & 0,2200 & 0,2547 & 6,6 \\
\hline 9 & 0,2642 & 0,2080 & 0,2824 & 12,6 \\
\hline 10 & 0,2613 & 0,2210 & 0,2617 & 7,7 \\
\hline 11 & 0,2763 & 0,1890 & 0,2874 & 17,5 \\
\hline 12 & 0,2851 & 0,3060 & 0,3008 & 3,0 \\
\hline 13 & 0,2555 & 0,2470 & 0,2644 & 2,8 \\
\hline 14 & 0,2579 & 0,2180 & 0,2647 & 8,3 \\
\hline 15 & 0,2773 & 0,2440 & 0,2828 & 6,4 \\
\hline 16 & 0,2634 & 0,2550 & 0,2910 & 5,7 \\
\hline 17 & 0,2692 & 0,3270 & 0,2739 & 9,0 \\
\hline 18 & 0,2657 & 0,2520 & 0,2741 & 3,4 \\
\hline 19 & 0,2517 & 0,2850 & 0,2647 & 5,1 \\
\hline 20 & 0,2515 & 0,2390 & 0,2538 & 2,6 \\
\hline 21 & 0,2655 & 0,2590 & 0,2831 & 3,8 \\
\hline 22 & 0,2668 & 0,2940 & 0,2785 & 4,0 \\
\hline 23 & 0,2241 & 0,2920 & 0,2210 & 13,3 \\
\hline 24 & 0,2565 & 0,2660 & 0,2699 & 2,1 \\
\hline 25 & 0,3329 & 0,3950 & 0,3246 & 9,0 \\
\hline 26 & 0,3137 & 0,3860 & 0,3324 & 8,9 \\
\hline 27 & 0,2537 & 0,2650 & 0,2617 & 1,8 \\
\hline 28 & 0,2282 & 0,2400 & 0,2422 & 2,6 \\
\hline 29 & 0,2351 & 0,2800 & 0,2357 & 8,4 \\
\hline
\end{tabular}

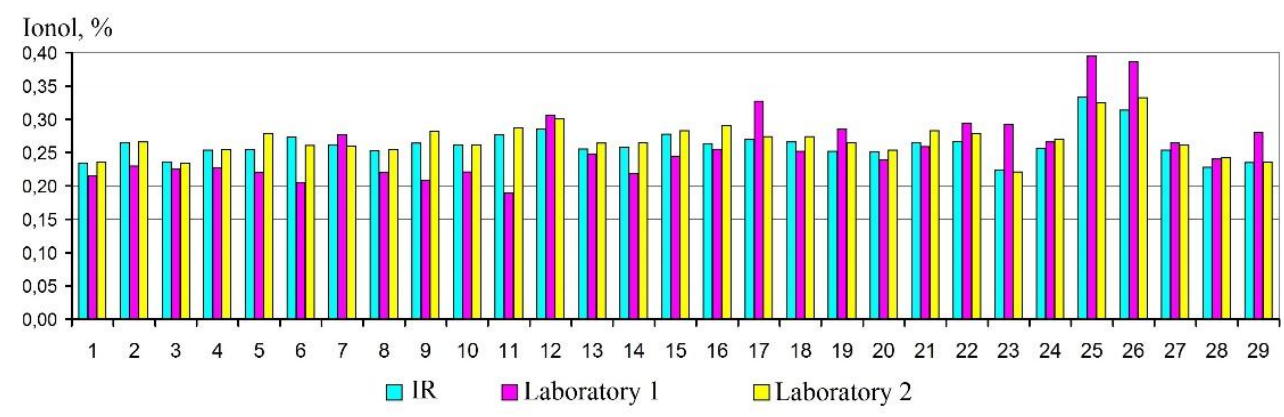

Figure 4. The results of ionol concentration measurement in oil samples No. $\div 29$ according to Table 1 . 


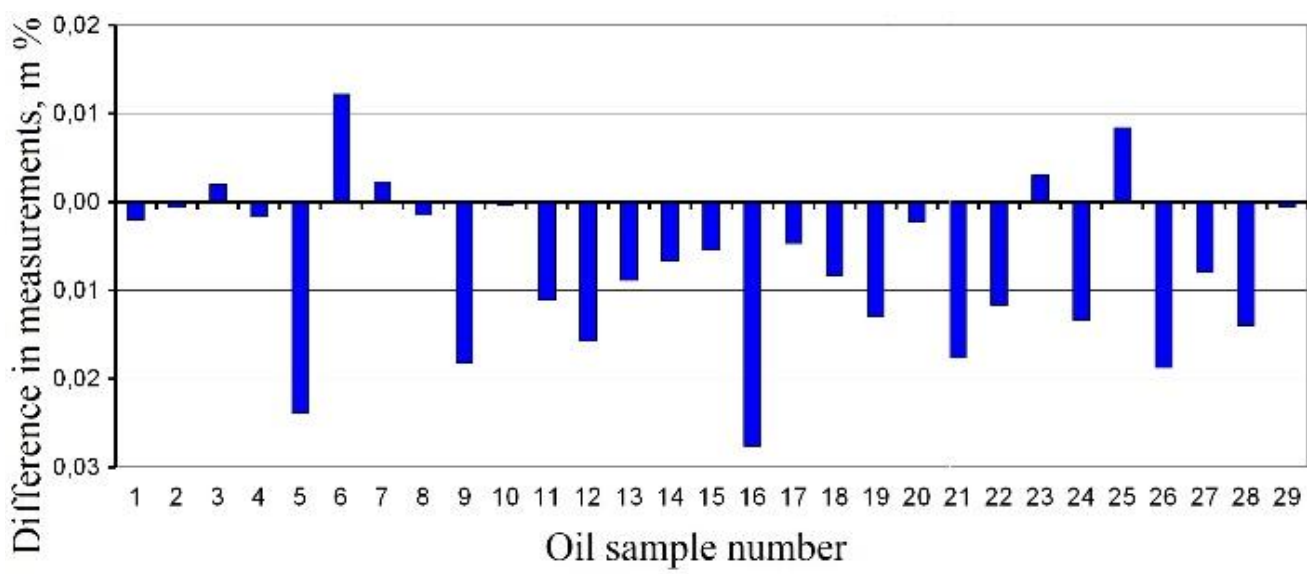

Figure 5. Difference in measurements of ionol concentration by gas chromatography in Laboratory 2 and IR spectroscopy.

A way to compare results obtained by different methods is to analyse differences in measurement results [14]. Fig. 5 shows the deviations of the results obtained by the IR method in comparison and GLC method (Laboratory 2).

The average value of the difference between both measurement methods was $-0.007 \%$ of ionol. The standard deviation of the differences was $0.009 \%$ of ionol. Such low values in comparison with the typically measured ionol concentrations (see Table 1) indicate the absence of a significant systematic discrepancy between both methods. We also concluded that there is no significant measurement error when using IR spectroscopy.

Thus, the comparison of the two measurement methods indicate the reliability the IR spectroscopy method and the possibility of practical use of this method for monitoring the ionol concentration in transformer oils.

Further, it is necessary to consider in more detail the above-mentioned problem of overestimating the results of the ionol concentration measurement by IR spectroscopy. This problem is due to the fact that decay products of the ionol molecule itself appear in the aging oil. According to modern concepts $[3,11,15,16]$, the proposed mechanism of ionol oxidation can be represented by the scheme shown in Fig. 6.
Typically 2,6-di-tert-butyl-phenol is indicated as the main compound accumulating in oils. Its structural formula is shown in the upper right corner of the diagram in Fig. 6. The presented compound, as well as other intermediate products of ionol decomposition, have the structure of sterically hindered phenols. Therefore, all these compounds, as well as the target component (ionol), will have an absorption band in the IR spectra in the region of $3650 \mathrm{~cm}^{-1}$, thereby distorting the measurement results.

As already noted, the problem of ionol degradation products accumulation in oil is rarely encountered in power transformers [11]. It should be noted that in accordance with Russian standard GOST 11677 oil transformers with a capacity of more than 1 MVA are equipped with thermosyphon or adsorption filters. As an adsorbent, as a rule, large-pore silica gel of the KSKG brand is used [17]. During the operation of the transformer, oil continuously circulates through the adsorbent layer, resulting in the precipitation of polar compounds in the pores of silica gel [18]. Such compounds, first of all, include various products of oxidation of hydrocarbon molecules (peroxides, alcohols, ketones, aldehydes, acids), as well as polyaromatic and resinous-asphaltene hydrocarbons.<smiles>CC(C)(C)C1=CC(=O)C=C(C(C)(C)C)C1=O</smiles>

Figure 6. The mechanism of oxidation of ionol in transformer oil [11] 
Shown in Fig. 6 intermediate products of degradation of the ionol molecule are also oxidized compounds of a polar nature. Based on this, we can assume that silica gel in adsorption and thermosyphon filters of power transformers can absorb such compounds. Only 2,6-ditert-butyl-phenol has the lowest polarity, which, like ionol, is practically not adsorbed by silica gel. However, with the removal of the intermediate products of ionol degradation from the oil, the problem of accumulation of this compound in the oil also ceases to be relevant.

Thus, based on the analysis of theoretical material and experimental data, we can conclude that the method of IR spectroscopy proposed in this article is promising for routine analyses of the ionol concentration in insulating oils. This method allows with high accuracy and reproducibility to obtain the results of the analysis of the additive content in oils from the power transformers equipped with adsorption cleaning systems. Those are all transformers with rated capacity of more than $1 \mathrm{MVA}$ installed in Russia.

In conclusion, it should be noted that in order to be able to apply the IR spectroscopy method to any types of insulating oils, it is necessary, in addition to the main absorption band $\left(3650 \mathrm{~cm}^{-1}\right)$, to use several additional bands, using the methods of multiple regression [19] and partial least squares [10]. However, this raises problems associated with the calibration of spectrometers, which casts doubt on the possibility of widespread use of this approach.

\section{Conclusion}

Based on the study of oils from power transformers, we showed that the method of IR spectroscopy and gasliquid chromatography give comparable results for determining the concentration of ionol. It is justified that when analysing oils from power transformers equipped with thermosyphon or adsorption filters, it is sufficient to use only one main absorption band of this additive to determine the ionol concentration.

\section{References}

1. J. Zou, W. Chen, F. Wan, Z. Fan, L Du, Energies, 9, 946 (2016).

2. P. Wiklund, B. Pahlavanpour, Journal of Iranian Association of Electrical and Electronics Engineers, 7, 65-73 (2010).

3. R.A. Lipshtejn, Proceedings of the Second AllUnion Scientific and Technical meetings "Oil Additives», 169-177 (1968).

4. Rosseti, The scope and standards of testing electrical equipment, 262 (2017).

5. IEC 60296 Fluids for electrotechnical applications Mineral insulating oils for electrical equipment: International standard, 82 (2020).

6. M.N. Lyutikova, A.A. Konovalov, S.M. Korobeinikov, V.K. Kozlov, M.Sh. Garifullin,
Power Technology and Engineering, 53 (1), 118-125 (2019).

7. STO 56947007-29.180.010.008-2008, Guidelines for the determination of ionol content in transformer oils by gas chromatography, 24 (2007).

8. IEC 60666 Detection and determination of specified additives in mineral insulating oils, 64 (2010).

9. ASTM D2668 Standard Test Method for 2,6-di-tertButyl-p-Cresol and 2,6-di-tert-Butyl Phenol in Electrical Insulating Oil by Infrared Absorption, 7, 6 (2013).

10. P. Ågren, T. Lillhonga, L. Melzer, 48 CIGRE Session, D1-103 (2020).

11. M.N. Lyutikova, S.V. Nehoroshev, V.M. Kuklina, M.G. Kul'kov, Power stations, 6, 47-53 (2020).

12. A. Smith, Applied IR Spectroscopy, 328 (2018).

13. S.M. Korobeinikov, M.N. Lyutikova, Problems of regional energy, 36, 96-105 (2018).

14. D. Tukey, Analysis of observation results. Exploratory analysis, 696 (1981).

15. R.A. Lipshtejn, M.I. Shahnovich, Transformer oil, 296 (1983).

16. V.A. Roginskij, Phenolic Antioxidants: Reactivity and Effectiveness, 247 (1988).

17. STO 70238424.27.100.053-2013, Power oils and oil facilities of power plants and networks. Organization of operation and maintenance. Norms and requirements, 163 (2013).

18. I.V. Braj, Regeneration of transformer oils, 168 (1972).

19. V.P. Krishhenko, Near infrared spectroscopy, 638 (1997). 